\title{
Risk factors correlated with antibiotic-induced carbapenem resistant in Gram-negative bacilli in China: a retrospective cohort study
}

\section{Jing Chen}

Sichuan Academy of Medical Sciences and Sichuan People's Hospital

\section{Qian Xiang}

Sichuan Academy of Medical Sciences and Sichuan Province People's Hospital

Jia-yu Wu

Sichuan Academy of Medical Science and Sichuan People's Hospital

Min-hong Cai

Sichuan Province People's Hospital

\section{Chen Wang}

Sichuan People's Hospital

\section{Dao-qiong Wei}

Sichuan Province People's Hosital

Hui Wang

Schuan Province People's Hospital

Yu Lv ( 29381636@qq.com )

https://orcid.org/0000-0002-8069-4801

\section{Research}

Keywords: carbapenem resistance; Gram-negative bacilli; quinolone

Posted Date: March 25th, 2020

DOI: https://doi.org/10.21203/rs.2.24013/v2

License: (c) (i) This work is licensed under a Creative Commons Attribution 4.0 International License. Read Full License 


\section{Abstract}

Background: Increasing resistance to carbapenem, particularly common in Gram-negative bacilli (GNB), has become a growing public health concern around the world. The objective of this study was to investigate risk factors associated with antibiotic-induced carbapenem resistant in Gram-negative bacilli (CR-GNB) among inpatients. Methods: A retrospective cohort study was conducted in one of the largest tertiary A-level hospitals including patients with GNB cultured from any of the clinical specimens who had been admitted for more than 2 calendar days from January 2017 to June 2019. Kaplan-Meier analysis and Cox proportional hazard model were used to estimate the hazard of CR-GNB induction by antibiotics. Results: 2490 patients including 7 cohorts were included. After cox proportional risk model analysis, carbapenems, $\beta$-lactamase inhibitors, and cephalosporins had significantly higher hazards than other types of antimicrobial $(P<0.001)$. But even without using any antimicrobials, the hazard would increase with the length of hospital stay. On multivariate analysis, carbapenem was the most principal hazard factor for antibiotic-induced CR-GNB (hazard ratio [HR], 2.968; 95\% confidence interval [Cl], 1.706ه5.162), followed by ICU admission (HR, 1.815; 95\% Cl, 1.507ه2.186), cephalosporin (HR, 1.605; 95\% Cl, 1.288区 1.999), tracheotomy (HR, 1.563; $95 \% \mathrm{Cl}, 1.251 \mathrm{1} 1.952)$ and $\beta$-lactamase inhibitor (HR, 1.542; $95 \% \mathrm{Cl}, 1.237 \rrbracket$ 1.921). However, quinolone effects on antibiotic-induced CR-GNB were not statistically significant. Conclusions: Prior carbapenem was a strong risk factor for antibiotic-induced CR-GNB, but quinolone was not associated with that. Rational use of carbapenems should be implemented and antimicrobial stewardship policies should be adjusted according to the characteristics of each hospital.

\section{Background}

Over recent years, antibiotic resistance has become an increasingly serious threat to public health. Multidrug resistance is especially common in Gram-negative bacilli (GNB), and the mortality, morbidity and cost burden are higher when infected with multidrug-resistant GNB [1-4].

Carbapenem is an atypical $\beta$ - lactam antibiotic with wide antibacterial spectrum and strong antibacterial activity which is often used as a last resort in the treatment of drug-resistant GNB infection [5]. However, the incidence of carbapenem-resistant bacteria has constantly increased over past years, posing a challenge for clinical antimicrobial chemotherapy and hospital infection control $[6,7]$.

In China, the four major Gram-negative bacterial species were Escherichia coli, Klebsiella pneumoniae, Acinetobacter baumannii and Pseudomonas aeruginosa according to CHINET surveillance in 2018 [8]. The resistance rate of $E$. coli, K. pneumoniae, $A$. baumannii and $P$. aeruginosa fluctuated around $2.0 \%-2.1 \%, 25 \%-26.3 \%, 77.1 \%-78.1 \%$ and $25.8 \%-30.7 \%$, respectively [8]. Previous studies had reported that carbapenem resistance may increase the mortality in patients with GNB infection [9-11].

Rational use of antibiotics can effectively treat bacterial infections and reduce the burden to patients. But unreasonable use of antibiotics will increase the selective pressure of antimicrobials commonly used, which is one of the important factors leading to antimicrobial resistance (AMR), and some evidence showed that increased use of antibiotics could lead to the emergence of AMR [11-17]. However, most 
related studies reported the significant relationship between carbapenem consumption and carbapenemresistant gram-negative bacilli (CR-GNB), and whether the use of other antibiotics contributes to CR-GNB is not yet certain. In addition, most of previous studies have focused on a certain type of CR-GNB, such as carbapenem-resistant P. aeruginosa (CRPA), while research on the whole CR-GNB is relatively insufficient. Accordingly, we conducted this study to assess the risk factors for isolation of antibiotic-induced CR-GNB in a large cohort.

\section{Materials And Methods}

\section{Design and subjects}

A retrospective cohort study was performed in one of the largest tertiary A-level hospitals in Sichuan Province, China from January 2017 through June 2019. The cohort study included all patients with gram negative bacilli (GNB) cultured from any of the clinical specimens who had been admitted for more than 2 calendar days. This study was approved by the Ethics Committee of Sichuan Academy of Medical Sciences and Sichuan Provincial People's Hospital. The Review Board exempted requirement for informed consent because of the retrospective study and absence negative impact on the patients.

\section{Cohorts}

This study included 7 cohorts, including cohort-Carbapenems, cohort- $\beta$-lactamase inhibitor, cohortAntifungal, cohort-Quinolones, cohort-Penicillins, cohort-Cephalosporins and cohort-Unused.

Cohort-Carbapenems: All patients with clinical samples positive for GNB who have only used carbapenem antibiotics before GNB was isolated.

Cohort- $\beta$-lactamase inhibitor: All patients with clinical samples positive for GNB who have only used $\beta$ lactamase inhibitor antibiotics before GNB was isolated.

Cohort-Antifungal: All patients with clinical samples positive for GNB who have only used antifungals before GNB was isolated.

Cohort-Quinolone: All patients with clinical samples positive for GNB who have only used quinolone antibiotics before GNB was isolated.

Cohort-Penicillin: All patients with clinical samples positive for GNB who have only used penicillins antibiotic before GNB was isolated.

Cohort-Cephalosporin: All patients with clinical samples positive for GNB who have only used cephalosporin antibiotics before GNB was isolated.

Cohort-Unused: All patients with clinical samples positive for GNB who have not used any antibiotic before GNB was isolated. 


\section{Outcomes and definition}

Antibiotic-induced CR-GNB was the primary outcome of this study.

Carbapenem resistant Gram-Negative Bacilli (CR-GNB): Carbapenem-resistance in GNB was defined as being non susceptible to imipenem, meropenem, ertapenem or doripenem. Species identification and in vitro susceptibility to imipenem, meropenem, ertapenem and doripenem were determined using Vitek-2 (BioMerieux, France) following the breakpoints defined by the Clinical Laboratory Standards Institute [18].

Antibiotic-induced CR-GNB: CR-GNB isolated after 2 calendar days of antimicrobial use.

\section{Confounding factors}

Potential confounders were selected based on previous literature [19-21], and included age, gender, principal diagnosis, tumor, liver failure, kidney failure, heart failure, respiratory failure, diabetes, hypertension, chronic obstructive pulmonary disease, hemodialysis, venous catheterization, mechanical ventilation, urinary catheterization, tracheotomy, surgery, ICU admission during current or previous episodes.

\section{Statistical analysis}

Statistical analysis of the data was performed using STATA 12.0. Data were summarized using the mean and standard deviation (SD) for normally distributed variables. Categorical variables were expressed in absolute numbers and percentages.

We used both univariate and multivariate methods to analyze the data. Binary outcomes were tested using the $\chi 2$ test, and continuous data were compared using the T-test. Kaplan-Meier analysis and Cox proportional hazard model were used to estimate the hazard of CR-GNB induction by antibiotics. $P$ values below 0.05 were considered significant.

\section{Results}

\section{Patient inclusion}

From January 1, 2017 to June 30, 2019, a total of 8748 inpatients with GNB isolated from any of the clinical specimens who were admitted, including 2587 patients with CRO isolated and 5417 patients with CSO isolated. After screening, 2490 patients including 7 cohorts were included in the final data set, as Fig. 1 shows.

\section{Factors associated with antibiotic-induced CR-GNB in univariate analysis}

Differences in antibiotic-induced CR-GNB effects between the 7 cohorts of antimicrobial drugs were statistically significant $(, P<0.001)$. In addition to antibacterial drugs, the effects of other factors on antibiotic-induced CR-GNB were also statistically significant, including gender, principal diagnosis, chronic 
obstructive pulmonary disease, venous catheterization, mechanical ventilation, urinary catheterization, tracheotomy, blood transfusion or use of blood products, surgery, kidney failure, respiratory failure, ICU admission now or in the past and community infections. Table 1 shows these details.

\section{Kaplan-Meier analysis}

Comparison of 7 cohorts showed that carbapenem, $\beta$-lactamase inhibitor, and cephalosporin had significantly higher hazards than any other type of antimicrobials. Even without the use of any antimicrobials, the hazard would increase with the length of hospital stay. Figure 2 shows these details. Log-rank test results showed that the differences were statistically significant (chi-square $=76.190, P<$ $0.001)$.

\section{Multivariate analysis}

The results of the COX proportional hazard model showed that carbapenem use was the principal hazard factor for antibiotic-induced CR-GNB (hazard ratio, 2.968; 95\% confidence interval, 1.706 $\$ 5.162$ ), followed by ICU admission now or in the past (hazard ratio, 1.815; 95\% confidence interval, 1.507ه2.186), cephalosporin (hazard ratio, 1.605; 95\% confidence interval, 1.288ه1.999), tracheotomy (hazard ratio, $1.563 ; 95 \%$ confidence interval, 1.251ه1.952) and $\beta$-lactamase inhibitor (hazard ratio, $1.542 ; 95 \%$ confidence interval, 1.237ه1.921). In addition, the hazard factors affecting antibiotic-induced CR-GNB included kidney failure, blood transfusion or use of blood products, gender, chronic obstructive pneumonia and principal diagnosis. But antifungal, quinolone, and penicillin effects on antibiotic-induced CR-GNB were not statistically significant. The details are shown in Table 2.

\section{Discussion}

Carbapenems are the most effective drugs for the treatment of severe infections with gram-negative bacteria due to their broad antimicrobial spectrum and high stability for hydrolysis by most $\beta$-lactamases, including extended-spectrum $\beta$-lactamases (ESBLs) and AmpC cephalosporinases [22]. But unreasonable use of antibiotics leads to the emergence of CR-GNB, which is becoming more and more serious, and the treatment of some drug-resistant infections is extremely limited $[8,23,24]$. Therefore, understanding the hazard factors for antibiotic-induced CR-GNB is very important in the early selection of empirical antibiotic program. We demonstrated that previous carbapenem exposure was the major risk factor for antibiotic-induced CR-GNB in our study population.

During the period, cephalosporins were the most commonly used antibiotics, followed by $\beta$-lactamase inhibitors and penicillins. Unlike this, physicians in Europe preferred to prescribe more penicillins than cephalosporins [25]. The difference might be explained by that most antibiotics were used for inpatient treatment in China while outpatient institutions accounted for the majority of antibiotic consumption in foreign countries [26-28]. The clinical application of carbapenem, as a special class antibiotic, is limited and requires pre-authorization during the using period in our country. 
In this study, carbapenem, $\beta$-lactamase inhibitor, and cephalosporin had significantly higher hazards than other types of antimicrobials. Our finding that carbapenem use was the most principal hazard factor associated with antibiotic-induced CR-GNB was in accordance with some previous studies [5, 29-35]. The use of carbapenem may promote the production of carbapenemase, such as K pneumoniae carbapenemase and metallo- $\beta$-lactamases, which could increase carbapenem-resistant Escherichia coli (CRE) [36]. Other mechanisms of carbapenem resistance include outer membrane porin expression loss combined with extended-spectrum $\beta$-lactamase (ESBL) and AmpC enzyme, change of antimicrobial target and high expression of efflux pump [37-39]. This suggested that controlling the use of carbapenems could slow down the production of CR-GNB. However, unlike some studies, we did not find that previous use of quinolones was significantly correlated with antibiotic-induced CR-GNB [29, 34, 35, 40-45]. It was speculated that quinolones might induce the high expression of efflux pump and lead to the multidrugresistant phenotype, probably CR-GNB [46-48]. The reason for this difference might be that our design or study population was different from previous studies. Another large-scale research in China showed that fluoroquinolone consumption was not associated with CR-GNB [5]. In one way, this may be a feature of our country, and could provide basis for our choice of anti-infective therapy. Although using antibiotic was an important risk factor for drug resistance, even without the use of any antimicrobials the hazard would increase with the length of hospital stay after cox model analysis. The result might be explained by that these patients with longer hospital stay had prolonged exposure to invasive devices or use of antibiotics.

ICU stay and tracheotomy were also important hazard factors associated with antibiotic-induced CR-GNB. ICU is considered as the main source of development of multidrug-resistant bacteria and transmission due to the extremely critically ill patients, the use of invasive devices and higher intensity of selection pressure by broad-spectrum antibiotics. When patients receive tracheotomy and ventilation, the normal upper respiratory barrier will be destroyed and the pipe will be gradually polluted by bacteria which will increase chance of bacterial invasion. Our results showed that the risk of antibiotic-induced CR-GNB isolation was 1.563 times higher in patients receiving tracheotomy than in patients without tracheotomy. This indicated that some preventive and control measures related to the use of tracheotomy, such as understanding the indication of tracheotomy, operation procedure and hand hygiene, could effectively reduce the CR-GNB in our hospital.

In our hospital, infection control measures against resistant bacteria are relatively comprehensive, including training for prevention and control methods for bacteria, active surveillance of the most common multidrug resistant bacteria (MDRO), computer system alerts for MDRO species, active screening for high risk inpatients, supervision of adherence to precaution measures and so on, to reduce the spread of MDROs in hospital.

There are several potential limitations in our study. Firstly, this study was a retrospective design conducted in a large tertiary A-level hospital, not a multicenter research. Secondly, we did not take into account the dose of the antibiotic and antibiotic combinations. Thirdly, the generation of CR-GNB could be varied by the practice of infection control and other risk factors, but we did not take the former into 
account. Despite these limitations, we believe that the main advantage of our research is its large cohort. Therefore, our research can at least provide reference value for the use of antibacterial drugs.

\section{Conclusion}

In conclusion, the use of carbapenem was strongly associated with antibiotic-induced CR-GNB in our study. Other antibiotics including cephalosporin and $\beta$-lactamase inhibitor were also related with CR-GNB. However, we did not find significant correlation between quinolones and antibiotic-induced CR-GNB. The findings will be useful for directing antimicrobial stewardship policies. Rational use of antibiotics and infection control measures are urgently needed to reduce the selective pressure of antibiotics, delay the occurrence of CR-GNB and control its cross transmission.

\section{Declarations}

\section{Ethics approval and consent to participate}

This study was approved by the Ethics Committee of Sichuan Academy of Medical Sciences and Sichuan Provincial People's Hospital. The Review Board exempted requirement for informed consent because of the retrospective study and no any negative impact on the patients.

\section{Consent for publication}

Not applicable.

\section{Availability of data and materials}

The datasets used and/or analysed during the current study are available from the corresponding author on reasonable request.

\section{Competing interests}

The authors declare that they have no competing interests.

\section{Funding}

Not applicable.

\section{Authors' contributions}

YL and JW designed the study. MC, HW, DW and CW collected the data. YL and QX performed the data analysis. JC and YL wrote the manuscript. All authors have read and critically revised the manuscript.

\section{Acknowledgements}

Not applicable. 


\section{References}

1. Chandy SJ, Naik GS, Balaji V, Jeyaseelan V, Thomas K, Lundborg CS: High cost burden and health consequences of antibiotic resistance: the price to pay. J Infect Dev Countr 2014, 8(9):1096-1102.

2. Giske CG, Monnet DL, Cars O, Carmeli Y, ReAct-Action on Antibiotic R: Clinical and economic impact of common multidrug-resistant gram-negative bacilli. Antimicrob Agents Chemother 2008, 52(3):813821.

3. Mauldin PD, Salgado CD, Hansen IS, Durup DT, Bosso JA: Attributable hospital cost and length of stay associated with health care-associated infections caused by antibiotic-resistant gram-negative bacteria. Antimicrob Agents Chemother 2010, 54(1):109-115.

4. Giraldi G, Montcsano M, Napoli C, Frati P, La Russa R, Santurro A, Scopetti M, Orsi GB: HealthcareAssociated Infections Due to Multidrug-Resistant Organisms: a Surveillance Study on Extra Hospital Stay and Direct Costs. Curr Pharm Biotechno 2019, 20(8):643-652.

5. Yang P, Chen YB, Jiang SP, Shen P, Lu XY, Xiao YH: Association between antibiotic consumption and the rate of carbapenem-resistant Gram-negative bacteria from China based on 153 tertiary hospitals data in 2014. Antimicrob Resist In 2018, 7.

6. . In: Guidelines for the Prevention and Control of Carbapenem-Resistant Enterobacteriaceae, Acinetobacter baumannii and Pseudomonas aeruginosa in Health Care Facilities. edn. Geneva; 2017.

7. Schwaber MJ, Carmeli Y: Carbapenem-resistant Enterobacteriaceae: a potential threat. JAMA 2008, 300(24):2911-2913.

8. Hu F, Guo Y, Yang Y, Zheng Y, Wu S, Jiang X, Zhu D, Wang F, China Antimicrobial Surveillance Network Study G: Resistance reported from China antimicrobial surveillance network (CHINET) in 2018. Eur J Clin Microbiol Infect Dis 2019, 38(12):2275-2281.

9. Lemos EV, de la Hoz FP, Einarson TR, McGhan WF, Quevedo E, Castaneda C, Kawai K: Carbapenem resistance and mortality in patients with Acinetobacter baumannii infection: systematic review and meta-analysis. Clin Microbiol Infect 2014, 20(5):416-423.

10. Xu L, Sun X, Ma X: Systematic review and meta-analysis of mortality of patients infected with carbapenem-resistant Klebsiella pneumoniae. Ann Clin Microbiol Antimicrob 2017, 16(1):18.

11. Liu Q, Li X, Li W, Du X, He JQ, Tao C, Feng Y: Influence of carbapenem resistance on mortality of patients with Pseudomonas aeruginosa infection: a meta-analysis. Sci Rep 2015, 5:11715.

12. Hsueh PR, Chen WH, Luh KT: Relationships between antimicrobial use and antimicrobial resistance in Gram-negative bacteria causing nosocomial infections from 1991-2003 at a university hospital in Taiwan. Int J Antimicrob Ag 2005, 26(6):463-472.

13. Chen IL, Lee CH, Su LH, Tang YF, Chang SJ, Liu JW: Antibiotic consumption and healthcareassociated infections caused by multidrug-resistant gram-negative bacilli at a large medical center in Taiwan from 2002 to 2009: implicating the importance of antibiotic stewardship. PLoS One 2013, 8(5):e65621. 
14. Tammer I, Geginat G, Lange S, Kropf S, Lodes U, Schluter D, Lippert H, Meyer F: [Antibiotic Consumption and the Development of Antibiotic Resistance in Surgical Units]. Zentralbl Chir 2016, 141(1):53-61.

15. Bell BG, Schellevis F, Stobberingh E, Goossens H, Pringle M: A systematic review and meta-analysis of the effects of antibiotic consumption on antibiotic resistance. BMC Infect Dis 2014, 14:13.

16. Mascarello M, Simonetti O, Knezevich A, Carniel LI, Monticelli J, Busetti M, Schincariol P, Torelli L, Luzzati R: Correlation between antibiotic consumption and resistance of bloodstream bacteria in a University Hospital in North Eastern Italy, 2008-2014. Infection 2017, 45(4):459-467.

17. Chaouch C, Hassairi A, Riba M, Boujaafar N: Association between bacterial resistance and antimicrobial consumption. Ann Biol Clin-Paris 2014, 72(5):555-560.

18. Clinical and Laboratory Standards Institute (2013) Performance Standards for Antimicrobial Susceptibility Testing. Twenty-Third Information Supplement (M100-S23). Clinical and Laboratory Standards Institute, Wayne.

19. Sultan AM, Seliem WA: Identifying Risk Factors for Healthcare-Associated Infections Caused by Carbapenem-Resistant Acinetobacter baumannii in a Neonatal Intensive Care Unit. Sultan Qaboos Univ Med J 2018, 18(1):e75-e80.

20. Ting SW, Lee CH, Liu JW: Risk factors and outcomes for the acquisition of carbapenem-resistant Gram-negative bacillus bacteremia: A retrospective propensity-matched case control study. J Microbiol Immunol Infect 2018, 51(5):621-628.

21. Marchenay P, Blasco G, Navellou JC, Leroy J, Cholley P, Talon D, Bertrand X, Gbaguidi-Haore H: Acquisition of carbapenem-resistant Gram-negative bacilli in intensive care unit: Predictors and molecular epidemiology. Med Maladies Infect 2015, 45(1-2):34-40.

22. Xia Y, Liang Z, Su X, Xiong Y: Characterization of carbapenemase genes in Enterobacteriaceae species exhibiting decreased susceptibility to carbapenems in a university hospital in Chongqing, China. Ann Lab Med 2012, 32(4):270-275.

23. Qin X, Yang Y, Hu F, Zhu D: Hospital clonal dissemination of Enterobacter aerogenes producing carbapenemase KPC-2 in a Chinese teaching hospital. J Med Microbiol 2014, 63(Pt 2):222-228.

24. Hu FP, Guo Y, Zhu DM, Wang F, Jiang XF, Xu YC, Zhang XJ, Zhang CX, Ji P, Xie Y et al: Resistance trends among clinical isolates in China reported from CHINET surveillance of bacterial resistance, 2005-2014. Clin Microbiol Infec 2016, 22:S9-S14.

25. de Sande-Bruinsma NV, Grundmann H, Verloo D, Tiemersma E, Monen J, Goossens $H$, Ferech M, Resistance EA, Antimicrobia ES: Antimicrobial Drug Use and Resistance in Europe. Emerging Infectious Diseases 2008, 14(11):1722-1730.

26. Lin H, Dyar OJ, Rosales-Klintz S, Zhang J, Tomson G, Hao M, Stalsby Lundborg C: Trends and patterns of antibiotic consumption in Shanghai municipality, China: a 6 year surveillance with sales records, 2009-14. J Antimicrob Chemother 2016, 71(6):1723-1729.

27. Adriaenssens N, Coenen S, Versporten A, Muller A, Minalu G, Faes C, Vankerckhoven V, Aerts M, Hens N, Molenberghs G et al: European Surveillance of Antimicrobial Consumption (ESAC): outpatient 
antibiotic use in Europe (1997-2009). J Antimicrob Chemother 2011, 66 Suppl 6:vi3-12.

28. Meyer E, Gastmeier P, Deja M, Schwab F: Antibiotic consumption and resistance: data from Europe and Germany. Int J Med Microbiol 2013, 303(6-7):388-395.

29. Ahn JY, Song JE, Kim MH, Choi H, Kim JK, Ann HW, Kim JH, Jeon Y, Jeong SJ, Kim SB et al: Risk factors for the acquisition of carbapenem-resistant Escherichia coli at a tertiary care center in South Korea: a matched case-control study. Am J Infect Control 2014, 42(6):621-625.

30. Ye JJ, Huang CT, Shie SS, Huang PY, Su LH, Chiu CH, Leu HS, Chiang PC: Multidrug resistant Acinetobacter baumannii: risk factors for appearance of imipenem resistant strains on patients formerly with susceptible strains. PLoS One 2010, 5(4):e9947.

31. Coppry M, Jeanne-Leroyer C, Noize P, Dumartin C, Boyer A, Bertrand X, Dubois V, Rogues AM: Antibiotics associated with acquisition of carbapenem-resistant Pseudomonas aeruginosa in ICUs: a multicentre nested case-case-control study. J Antimicrob Chemother 2019, 74(2):503-510.

32. Hu Y, Ping Y, Li L, Xu H, Yan X, Dai H: A retrospective study of risk factors for carbapenem-resistant Klebsiella pneumoniae acquisition among ICU patients. J Infect Dev Ctries 2016, 10(3):208-213.

33. Cao J, Song W, Gu B, Mei YN, Tang JP, Meng L, Yang CQ, Wang HJ, Zhou H: Correlation Between Carbapenem Consumption and Antimicrobial Resistance Rates of Acinetobacter baumannii in a University-Affiliated Hospital in China. J Clin Pharmacol 2013, 53(1):96-102.

34. in 't Holt AFV, Severin JA, Lesaffre EMEH, Vos MC: A Systematic Review and Meta- Analyses Show that Carbapenem Use and Medical Devices Are the Leading Risk Factors for CarbapenemResistant Pseudomonas aeruginosa. Antimicrob Agents Ch 2014, 58(5):2626-2637.

35. Liu P, Li X, Luo M, Xu X, Su K, Chen S, Qing Y, Li Y, Qiu J: Risk Factors for Carbapenem-Resistant Klebsiella pneumoniae Infection: A Meta-Analysis. Microb Drug Resist 2018, 24(2):190-198.

36. Navon-Venezia S, Chmelnitsky I, Leavitt A, Schwaber MJ, Schwartz D, Carmeli Y: Plasmid-mediated imipenem-hydrolyzing enzyme KPC-2 among multiple carbapenem-resistant Escherichia coli clones in Israel. Antimicrob Agents Ch 2006, 50(9):3098-3101.

37. Santajit S, Indrawattana N: Mechanisms of Antimicrobial Resistance in ESKAPE Pathogens. Biomed Res Int 2016, 2016:2475067.

38. Nordmann P, Dortet L, Poirel L: Carbapenem resistance in Enterobacteriaceae: here is the storm! Trends Mol Med 2012, 18(5):263-272.

39. Poirel L, Heritier C, Spicq C, Nordmann P: In vivo acquisition of high-level resistance to imipenem in Escherichia coli. J Clin Microbiol 2004, 42(8):3831-3833.

40. Montero M, Sala M, Riu M, Belvis F, Salvado M, Grau S, Horcajada JP, Alvarez-Lerma F, Terradas R, Orozco-Levi $\mathrm{M}$ et al: Risk factors for multidrug-resistant Pseudomonas aeruginosa acquisition. Impact of antibiotic use in a double case-control study. Eur J Clin Microbiol Infect Dis 2010, 29(3):335-339.

41. Lautenbach E, Weiner MG, Nachamkin I, Bilker WB, Sheridan A, Fishman NO: Imipenem resistance among Pseudomonas aeruginosa isolates: Risk factors for infection and impact of resistance on clinical and economic outcomes. Infect Cont Hosp Ep 2006, 27(9):893-900. 
42. Lesho EP, Clifford RJ, Chukwuma U, Kwak YI, Maneval M, Neumann C, Xie S, Nielsen LE, Julius MD, McGann P et al: Carbapenem-resistant Enterobacteriaceae and the correlation between carbapenem and fluoroquinolone usage and resistance in the US military health system. Diagn Microbiol Infect Dis 2015, 81(2):119-125.

43. Gasink LB, Edelstein PH, Lautenbach E, Synnestvedt M, Fishman NO: Risk Factors and Clinical Impact of Klebsiella pneumoniae Carbapenemase-Producing K. pneumoniae. Infect Cont Hosp Ep 2009, 30(12):1180-1185.

44. Tan CK, Tang HJ, Lai CC, Chen YY, Chang PC, Liu WL: Correlation between antibiotic consumption and carbapenem-resistant Acinetobacter baumannii causing health care-associated infections at a hospital from 2005 to 2010. J Microbiol Immunol 2015, 48(5):540-544.

45. Schwaber MJ, Klarfeld-Lidji S, Navon-Venezia S, Schwartz D, Leavitt A, Carmeli Y: Predictors of carbapenem-resistant Klebsiella pneumoniae acquisition among hospitalized adults and effect of acquisition on mortality. Antimicrob Agents Ch 2008, 52(3):1028-1033.

46. Alyaseen SA, Piper KE, Rouse MS, Steckelberg JM, Patel R: Selection of cross-resistance following exposure of Pseudomonas aeruginosa clinical isolates to ciprofloxacin or cefepime. Antimicrob Agents Chemother 2005, 49(6):2543-2545.

47. Terzi HA, Kulah C, Ciftci IH: The effects of active efflux pumps on antibiotic resistance in Pseudomonas aeruginosa. World J Microbiol Biotechnol 2014, 30(10):2681-2687.

48. Higgins PG, Wisplinghoff $H$, Stefanik D, Seifert $H$ : Selection of topoisomerase mutations and overexpression of adeB mRNA transcripts during an outbreak of Acinetobacter baumannii. J Antimicrob Chemother 2004, 54(4):821-823.

\section{Tables}

Table 1. Baseline characteristics 


\begin{tabular}{|c|c|c|c|c|}
\hline Variables & $\begin{array}{c}\text { CSO } \\
(n=1838)\end{array}$ & $\begin{array}{c}\text { CRO } \\
(n=652) \\
\end{array}$ & Statistics & $P$ \\
\hline Antibacterial drugs & & & 80.051 & 0.000 \\
\hline Unused & $998(54.30 \%)$ & $257(39.42 \%)$ & & \\
\hline Carbapenem & $8(0.44 \%)$ & $14(2.15 \%)$ & & \\
\hline$\beta$-lactamase inhibitor & $216(11.75 \%)$ & $144(22.09 \%)$ & & \\
\hline Antifungal & $34(1.85 \%)$ & $14(2.15 \%)$ & & \\
\hline Quinolone & $74(4.03 \%)$ & $26(3.99 \%)$ & & \\
\hline Penicillin & $188(10.23 \%)$ & $54(8.28 \%)$ & & \\
\hline Cephalosporin & $320(17.41 \%)$ & $143(21.93 \%)$ & & \\
\hline Gender & $1061(57.73 \%)$ & $480(73.62 \%)$ & 51.544 & 0.000 \\
\hline Urinary cannula & $717(39.01 \%)$ & $331(50.77 \%)$ & 27.294 & 0.000 \\
\hline Vascular cannula & $303(16.49 \%)$ & $176(26.99 \%)$ & 34.208 & 0.000 \\
\hline Mechanical Ventilation & $230(12.51 \%)$ & $189(28.99 \%)$ & 93.326 & 0.000 \\
\hline Tracheotomy & $114(6.20 \%)$ & $112(17.18 \%)$ & 70.252 & 0.000 \\
\hline ICU admission now or in the past & $388(21.11 \%)$ & $253(38.80 \%)$ & 78.820 & 0.000 \\
\hline Hemodialysis & $5(0.27 \%)$ & $6(0.92 \%)$ & 3.242 & 0.072 \\
\hline Blood transfusion or use of blood products & $382(20.78 \%)$ & $235(36.04 \%)$ & 60.124 & 0.000 \\
\hline Hypertension & $873(47.50 \%)$ & $333(51.07 \%)$ & 2.465 & 0.116 \\
\hline Diabetes & $454(24.70 \%)$ & $157(24.08 \%)$ & 0.100 & 0.752 \\
\hline Chronic obstructive pneumonia & $203(11.04 \%)$ & $111(17.02 \%)$ & 15.617 & 0.000 \\
\hline Tumor & $269(14.64 \%)$ & $84(12.88 \%)$ & 1.214 & 0.270 \\
\hline Liver failure & $12(0.65 \%)$ & $9(1.38 \%)$ & 3.046 & 0.081 \\
\hline Kidney failure & $138(7.51 \%)$ & $77(11.81 \%)$ & 11.289 & 0.001 \\
\hline
\end{tabular}


Certain infectious diseases and parasites(A00-B99)

Tumor(C00-D48)

Blood and hematopoietic diseases and certain diseases involving immune mechanisms(D50-D89)

Endocrine, nutritional and metabolic diseases(E00-E90)

Mental and behavioral disorders(F00-F99)

Nervous system diseases(G00-G99)

Eye and appendage diseases(H00-H59)

Ear and mastoid diseases(H60-H95)

Circulatory diseases(I00-I99)

Respiratory diseases(J00-J99)

Digestive diseases(K00-K93)

Skin and subcutaneous tissue diseases(L00-L99)

Musculoskeletal system and connective tissue diseases(M00-M99)

Genitourinary diseases(N00-N99)

Pregnancy, childbirth and puerperium(O00-099)

Diseases that originated in the perinatal period(P00-P96)

Congenital malformations, deformation and chromosomal abnormalities(Q00-Q99)

Abnormal symptoms, signs, clinical and laboratory results, and cannot be classified in other categories (R00-R99)
$52(2.83 \%) \quad 20(3.07 \%)$

$202(10.99 \%) \quad 60(9.20 \%)$

$4(0.22 \%) \quad 4(0.61 \%)$

$56(3.05 \%) \quad 4(0.61 \%)$

$63(3.43 \%) \quad 15(2.30 \%)$

$149(8.11 \%) \quad 71(10.89 \%)$

$2(0.11 \%) \quad 0(0.00 \%)$

$3(0.16 \%) \quad 2(0.31 \%)$

$434(23.61 \%) \quad 171(26.23 \%)$

$270(14.69 \%) \quad 152(23.47 \%)$

$101(5.5 \%) \quad 19(2.91 \%)$

$38(2.07 \%) \quad 9(1.38 \%)$

$76(4.13 \%) \quad 6(0.92 \%)$

$153(8.32 \%) \quad 28(4.29 \%)$

$12(0.65 \%) \quad 0(0.00 \%)$

$5(0.27 \%) \quad 12(1.84 \%)$

$9(0.49 \%) \quad 3(0.46 \%)$

$15(0.82 \%) \quad 6(0.92 \%)$ 
Injury, poisoning and other external pathogenic factors (S00-T98)

External causes of illness and death(V01-V98)
$148(8.05 \%) \quad 60(9.20 \%)$

$46(2.50 \%) \quad 9(1.38 \%)$

$68.27 \pm 20.53 \quad 69.65 \pm 20.98$

$-1.473$

0.141

Table 2. Equation parameters of Cox proportional hazard model 
Antibacterial drugs

Carbapenem

$\beta$-lactamase inhibitor

Antifungal

Quinolone

Penicillin

Cephalosporin

Kidney failure

ICU admission now or in the past

Blood transfusion or use of blood products

Tracheotomy

Gender

Chronic obstructive pneumonia

Principal diagnosis(ICU-10 code)

Tumor(C00-D48)

Blood and hematopoietic diseases and certain diseases involving immune mechanisms(D50D89)

Endocrine, nutritional and metabolic diseases(E00-E90)

Mental and behavioral disorders(F00-F99)

Nervous system diseases(G00-G99)

Eye and appendage diseases(H00-H59)

Ear and mastoid diseases(H60-H95)

Circulatory diseases(I00-I99)
2.9681 .7065 .162

1.5421 .2371 .921

$1.107 .627 \quad 1.956$

$1.300 \quad 865 \quad 1.953$

$1.139 .842 \quad 1.541$

1.6051 .2881 .999

1.3601 .0591 .746

1.8151 .5072 .186

1.2881 .0781 .539

1.5631 .2511 .952

1.5311 .2781 .834

1.2731 .0071 .610

$.541 \quad .321 \quad .912$

$\begin{array}{lll}2.478 & 827 \quad 7.428\end{array}$

$\begin{array}{lll}.435 & 147 \quad 1.284\end{array}$

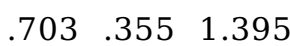

$\begin{array}{lll}1.050 .628 & 1.757\end{array}$ 
Respiratory diseases(J00-J99)

Digestive diseases(K00-K93)

Skin and subcutaneous tissue diseases(L00-L99)

Pregnancy, childbirth and puerperium(O00-O99)

Diseases that originated in the perinatal period(P00-P96) categories (R00-R99)

\section{Figures}

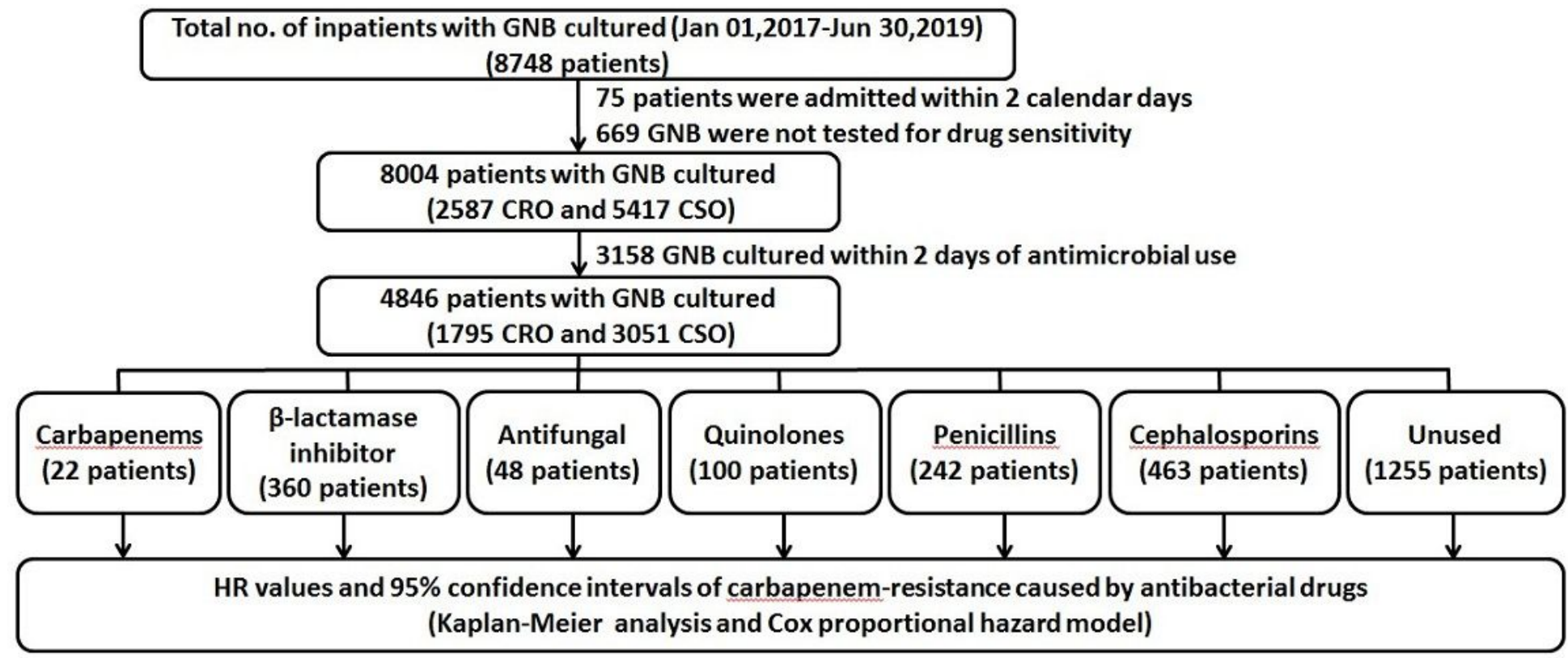

Figure 1 


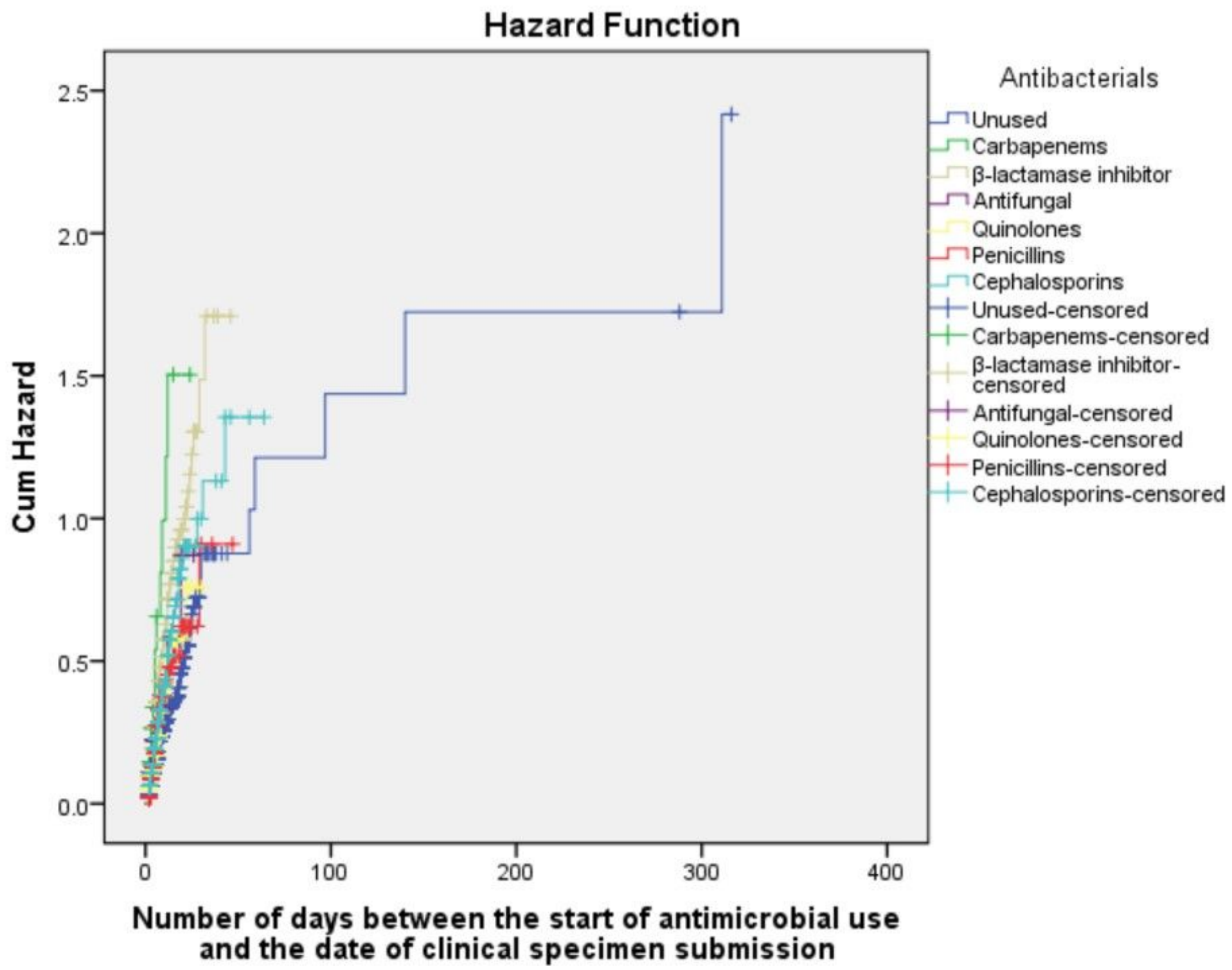

Figure 2

Results of Kaplan-Meier analysis 